\title{
Solar Wind Triggering of Substorm Expansion Onset
}

\author{
R. L. McPherron*, T. Terasawa, and A. Nishida \\ Institute of Space and Astronautical Science, 6-1 Komba 4-chome, Meguro-ku, Tokyo, Japan
}

(Received February 4, 1986; Revised May 7, 1986)

\begin{abstract}
Recent reports claim that the onset of the expansion phase of magnetospheric substorms is triggered by a sudden northward turning of the interplanetary magnetic field vector (IMF). We investigate this claim by studying the relation between sudden onsets of magnetic activity ( $A L$ index) and changes in the IMF (rectified electric field). Specific examples of the relation between the solar wind and magnetic activity demonstrate that sharp onsets in $A L$ occasionally appear to be independent of changes in the solar wind, but more frequently they appear to be triggered by northward turnings. A statistical analysis of this relation for the first six months of 1978 shows that auroral zone magnetic disturbance is present during approximately $73 \%$ of all hours. Distinct substorms defined by minima in the $A L$ index can be identified in $23 \%$ of these disturbed hours. Sharp onsets in $A L$ occur in about $45 \%$ of the identified substorms. For the subset of substorms with sharp onsets and corresponding solar wind data, $28 \%$ occur during steady southward IMF. However, a larger fraction of the sharp onsets (44\%) appears to be correlated with northward turnings of the IMF.

Because a significant number of sharp substorm expansion onsets occur during steady solar wind conditions, we conclude that the onset is caused by a process internal to the magnetosphere. However, since many other sharp onsets appear to be correlated with changes in either the IMF orientation or the solar wind dynamic pressure, we conclude this internal process can be influenced by changes in solar wind stress applied to the magnetosphere.
\end{abstract}

\section{Introduction}

The concept of a magnetospheric substorm (CORONITI et al., 1968) was originally introduced to model the phenomena which occur throughout the magnetosphere at the time of an auroral and polar magnetic substorm (AKASOFU, 1968). In the first model, the substorm began with of an auroral breakup and ended after the aurora recovered their quiet forms. The beginning of breakup was called the onset of the expansion phase. In a later modification, a growth phase was added prior to the onset of the expansion phase (MCPHERRON, 1970). It has recently become apparent that the magnetic perturbations of the growth phase are primarily caused by a global ionospheric current system made up of eastward and westward electrojets linked to a three-dimensional magnetospheric current system. Perturbations in the expansion phase are caused by a superposition of this global system and a more transient, three-dimensional current system localized near midnight (BAKER et al., 1984).

*Permanent address: Institute of Geophysics and Planetary Physics, University of California, Los Angeles California 90024, U.S.A. 
In one qualitative model of the magnetospheric substorm (RUSSELL and MCPHERRON, 1972; MCPHERRON et al., 1973) the expansion phase current system is caused by the diversion of tail current through the midnight ionosphere and is associated with the formation of a near-earth neutral line (NISHIDA and NAGAYAMA, 1973). Why a neutral line should form in this region is not understood, but the growth phase model suggests that it is a result of progressive changes in magnetospheric configuration brought about by unbalanced flux transfer between the day and night sides (MCPHERRON, 1979). In essence the tail current is diverted and a near-earth neutral line forms to rapidly return flux stripped from the day side by a southward directed interplanetary magnetic field (IMF).

In this model one would naively expect that a northward turning of the IMF would end flux transport to the tail and that magnetospheric convection would gradually decrease. Three-dimensional current systems driven by this convection would vanish as would their magnetic perturbations. However, there is a growing body of evidence that northward turnings of the IMF do not produce a gradual decay of magnetic activity. On the contrary, activity frequently increases at the time of such events. Furthermore, the activity at this time appears to have been triggered by the change in IMF as illustrated schematically in Fig. 1.

The possibility that directional discontinuities in the IMF trigger substorms was first discussed by BURCH (1973). The fact that the $B_{z}$ component plays a role in such triggering was noted by CAAN et al. (1975), who found statistically that major midlatitude positive bays and changes in magnetotail field strength followed a northward turning of the IMF. The authors later verified this result by an examination of case histories (CAAN et al., 1977). Since this work several other authors have reached similar conclusions (PELLINEN et al., 1982; ROSTOKER et al., 1982; ROSTOKER, 1983; ROSTOKER et al., 1983, 1984). Most recently, TROSCHICHEV et al. (1986), have reported that the probability of triggering by a northward turning is much enhanced in winter season if the $B_{y}$ component is negative.

Our work was motivated by a desire to establish how frequently a northward turning of the IMF triggers a substorm expansion. As we will show, the auroral zone magnetic records are disturbed most of the time. During much of this time no substorm can be readily identified. Even when substorms as defined by ROSTOKER et al. (1980) are present, the $A L$ index used in our study is often not sufficient to determine the time of expansion onset. However, a substantial number of substorms do have sharp onsets, and these may be compared to solar wind records of dynamic pressure and rectified electric field (IMF orientation). We find that a substantial fraction of all substorms with sudden onsets appears to be triggered by the IMF.

\section{Data Analysis Procedure}

As discussed above, the primary purpose of this work is to determine how often a substorm expansion is triggered by a northward turning of the interplanetary magnetic field. To obtain the times of substorm expansion onsets we make use of the $A L$ index. At any instant of time, this index is defined as the maximum negative perturbation in the horizontal component of the magnetic field observed anywhere in the auroral zone. This perturbation is caused by one of two current systems, the global convection system in the post midnight sector, or the substorm current wedge in the 
midnight sector. In a typical isolated substorm, slow changes in this index are caused by variations in magnetospheric convection, while sudden changes are caused by the formation and movement of the substorm current wedge. The formation of this wedge is coincident with the breakup and westward/poleward expansion of auroral arcs near midnight. For our purposes we define substorm onset as a sudden change in the slope of the graph of the $A L$ index versus time. Plots of this index are available from the World Data Center for Geomagnetism (KAMEI and MAEDA, 1981). A typical plot is reproduced in the top panel of Fig. 2.

In the first phase of our analysis we visually scanned plots of $A L$ for the first six months of 1978 and recorded the number of disturbed hours, the number of substorms, and the number of sharp onsets. A given hour was designated as disturbed if the minimum value of $A L$ in the hour was less than about $-200 \mathrm{nT}$. A substorm was recorded in each disturbed interval when the $A L$ index displayed a substantial minimum. To decide whether such a minima had occurred, we visually low-pass filtered the $A L$ index with about a one-hour time constant and recorded the hour in which the minima occurred. Finally, a sharp onset was defined as the beginning of any sudden decrease in the $A L$ index of order $100 \mathrm{nT}$ or larger.

While these definitions are quite subjective, they are usually easy to apply, as can be seen by examination of Fig. 2. On the day shown, there were ten quiet hours, three substorms ( 0600,1500 , and 2100 UT), and two sharp onsets ( 0640 and 2040 UT). The substorm beginning after 1100 and ending at 1700 UT had no sharp onset by our definition. The activity between 1700 and 2000 UT is relatively constant on a time scale of one hour and had no identifiable minima, so it was not classified as a distinct substorm. The times 0640 and 2040 UT clearly correspond to sudden changes in slope of the $A L$ index.

It should be emphasized that there is no reason to assume that the absence of sharp onsets in $A L$ during a disturbed interval implies there was no expansion onset. It is likely that many such onsets cannot be identified because magnetic observatories are not located at appropriate locations, or because the convectional electrojet is stronger than the substorm electrojet and hence determines the value of $A L$. Also, we have restricted ourselves to major onsets and have made no attempt to determine times of other onsets or intensifications in the same substorm. Our list of sharp onsets must be considered a lower limit to the number of onsets that actually occurred.

In the second phase of the analysis we scanned plots of five-minute averages of solar wind data produced by the National Space Science Data Center. An example of such data is presented in the bottom two panels of Fig. 2. The middle panel displays a logarithmic plot of the rectified solar wind electric field (product of solar wind velocity and the southward component of the IMF in GSM coordinates). The bottom panel displays a logarithmic plot of the solar wind dynamic pressure $\left(\mathrm{mnV}^{2}\right)$. Our procedure was to characterize both parameters at the time of sudden onsets in the $A L$ index. We also noted times of northward turnings and northward fluctuations of the IMF regardless of whether an $A L$ index onset had been recorded.

The results of this classification are summarized by the notations in the two bottom panels of Fig. 2. Northward turnings (NOTU) occurred at about 0240 and 1510 UT, while a northward fluctuation occurred at 2040 UT. For the onset which occurred at 0640 UT, the IMF was steadily northward (STNO). The bottom panel indicates that a sudden increase in dynamic pressure (PRIN) occurred in coincidence 


\section{A Search For The Causes Of Substorm Expansion Onsets}
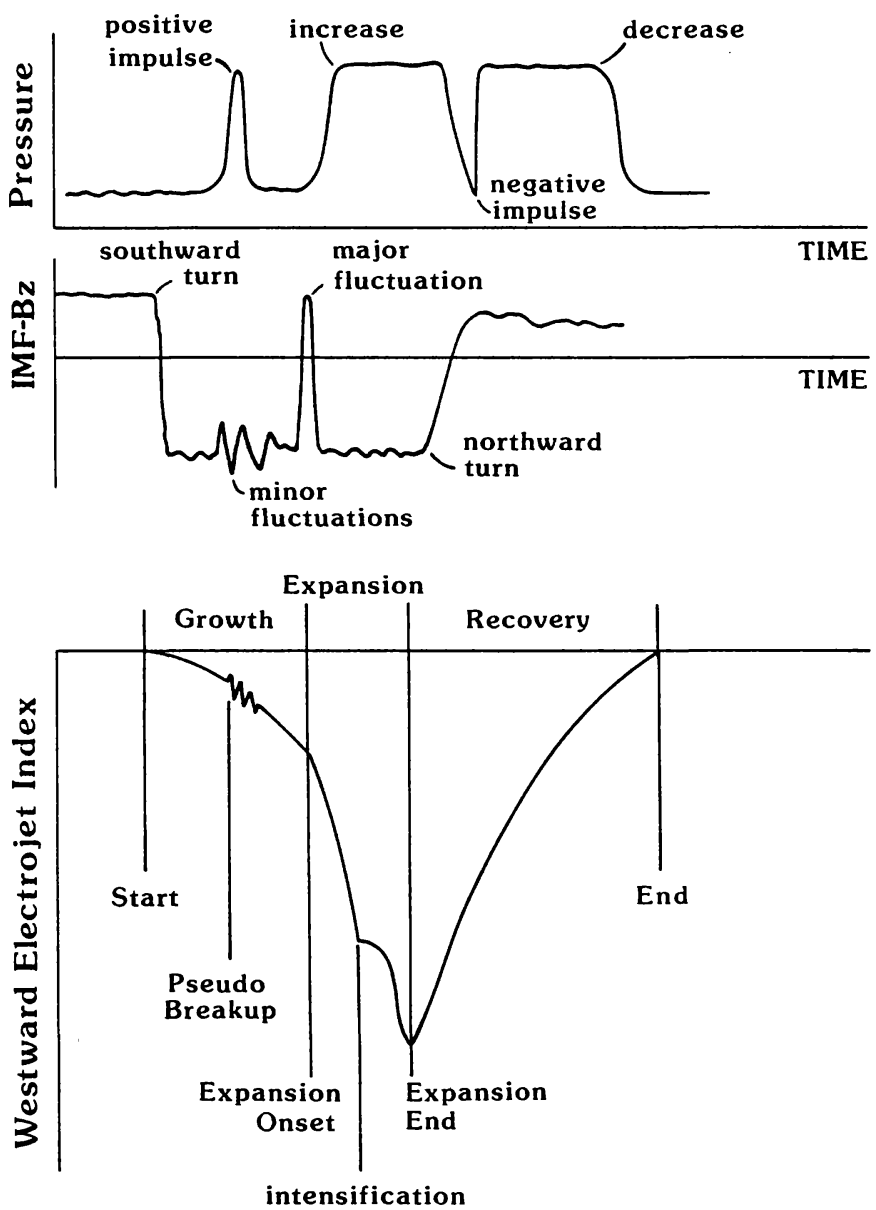

Fig. 1. Schematic relationship between solar wind and interplanetary magnetic field (IMF), and auroral zone magnetic activity. Top panel illustrates changes in solar wind dynamic pressure which might trigger substorm onset. Middle panel shows the same for IMF. Bottom panel displays typical variations in the westward electrojet index $(A L)$ during isolated substorm and defines substorm terminology.

with the 2040 UT northward fluctuation. A subsequent pressure decrease (PRDE) occurred at $2330 \mathrm{UT}$.

Further examination of the data in Fig. 2 reveals difficulties with our correlation procedure. For example, the southward IMF and northward turning between 0000 and 0300 UT produced no observable effect in $A L$. This is probably due to the weak nature of the electric field $(1 \mathrm{mV} / \mathrm{m})$ and the fact that any substorm activity would be 


\section{EXAMPLE OF DATA CLASSIFICATION}
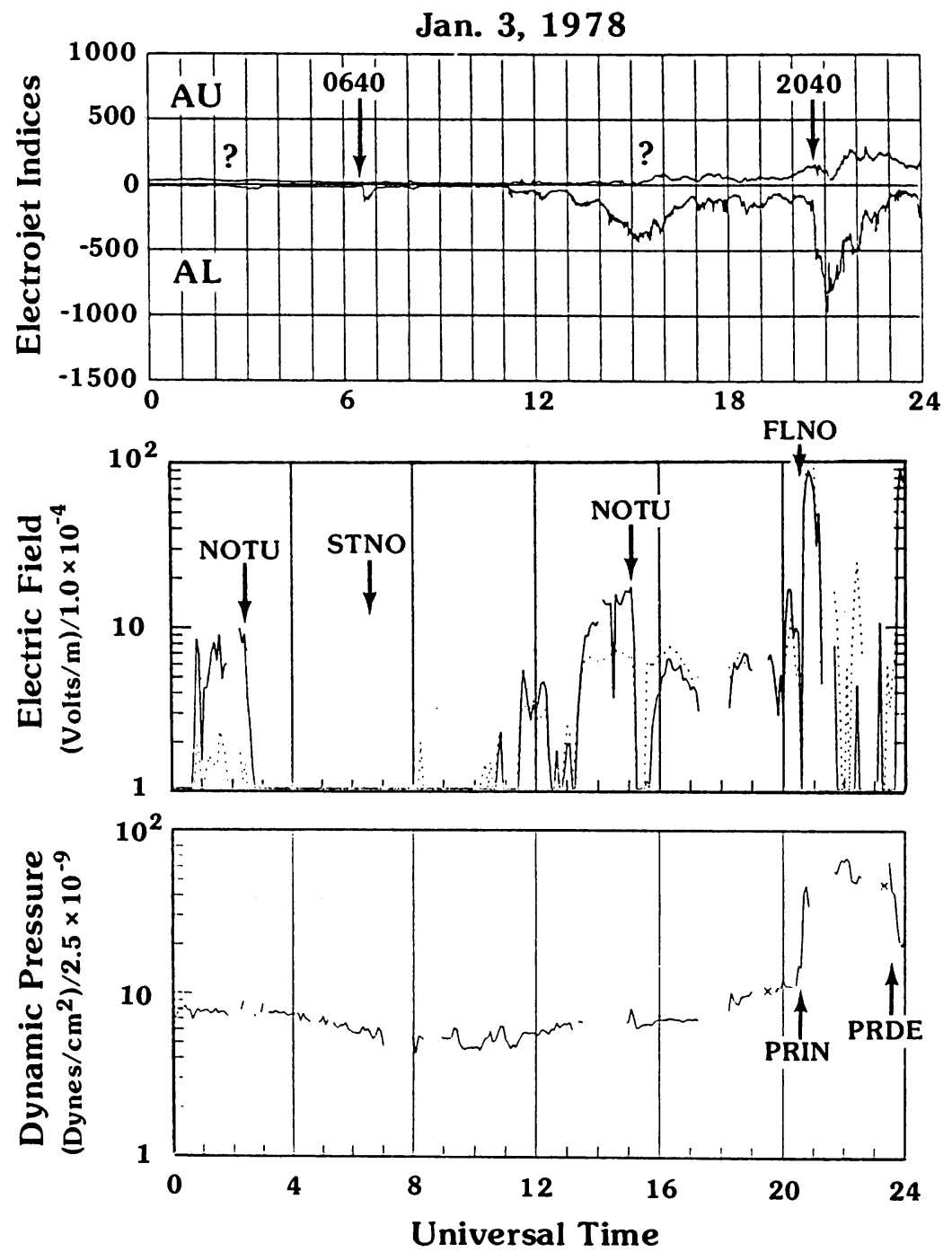

Fig. 2. Example of raw data used to generate statistics on the occurrence of solar wind triggered substorm onsets. Top panel shows the $A U$ and $A L$ index for a 24 hour interval. Middle panel shows a logarithmic plot of the rectified solar of the solar wind electric field. Bottom panel shows a logarithmic plot of the solar wind dynamic pressure. A sharp onset in $A L$ at 2040 UT appears to have been triggered by a northward turning of the IMF (electric field goes to zero) and an increase in dynamic pressure.

centered in the Atlantic Ocean where there are few observatories. Another example is the absence of any solar wind change at the time of the 0640 UT onset. One possible explanation for this is that the onset is caused by a delayed release of energy accumulated in the earlier episode of southward IMF. Yet another difficulty is the presence of simultaneous changes in all solar wind parameters which makes it 
impossible to decide what might be the cause of a sharp onset. A final difficulty is a fluctuating IMF, such as that recorded at the end of the day. In such cases it is impossible to decide whether any particular change causes an $A L$ onset. With these difficulties in mind, our summary statement for this day is that one sharp onset may have been triggered by an increase in solar wind dynamic pressure.

Altogether we scanned six months of data of the type shown in Fig. 2, characterizing the solar wind at the time of sharp $A L$ onsets, and the $A L$ index at the time of northward turnings. From this scan we identified a number of categories of relations between magnetic activity and the solar wind. From the events in each category we then selected particularly good examples for which we made higher resolution plots from digital data. These events are discussed in the following section.

\section{Examples of Relation between Solar Wind and Substorm Onset}

For the purposes of this paper, three types of solar wind-magnetic activity relations are particularly relevant, and we illustrate them here with specific examples. These categories include onsets apparently triggered by northward turnings, northward turnings that do not trigger onsets, and onsets without associated northward turnings.

\subsection{Onsets apparently triggered by northward turnings of the IMF}

Our first example of apparent triggering is presented in Fig. 3. The top panel shows six hours of data centered on the time of the sharp onset in the $A L$ index (vertical line). The top trace in this panel is the solar wind dynamic pressure, and the bottom trace is the rectified solar wind electric field. The latter is calculated from the product of the solar wind velocity and the magnitude of $B_{z}$ whenever it is negative (southward). Measures of the strength of the eastward and westward electrojets, the $A U$ and $A L$ indices, are displayed in the bottom panel.

For the event shown in Fig. 3, the IMF turned briefly southward about 2.5 hours before the $A L$ onset. It then turned northward for a short time and turned southward again at -1.5 hours. Five minutes before onset the IMF turned northward and remained there for at least 30 minutes; after this time no data are available in the solar wind. The $A U$ and $A L$ indices both begin to respond soon after the second southward turning; however, the main response in $A L$ follows the northward turning.

For this event we have made a detailed study of whether the onset satisfies causality, i.e., whether the IMF discontinuity causing the northward turning could have arrived at the magnetopause before the onset. The northward turning began slowly at $0710 \mathrm{UT}$, but by 0715 UT was rotating rapidly and reached a steady positive value by $0720 \mathrm{UT}$. At this time the IMP 8 spacecraft was located in GSM coordinates near $(26.7,-3.8,-9.2) \mathrm{Re}$, and the solar wind velocity was $303 \mathrm{~km} / \mathrm{s}$. If the discontinuity was convected radially earthward by the solar wind, and the normal to the discontinuity was along the earth sun line, then the disturbance should have reached the magnetopause at 0723 UT (allowing four minutes in the magnetosheath). An examination of auroral zone and midlatitude magnetograms from the midnight sector shows that the $A L$ onset occurred at 0722 UT. These times agree within the plus or minus 5-minute resolution of our solar wind data and we therefore conclude that the expansion onset could have been triggered. 


\section{COMPARISON OF SOLAR WIND INPUT WITH AURORAL ZONE MAGNETIC OUTPUT 07:20 UT February 12, 1978}
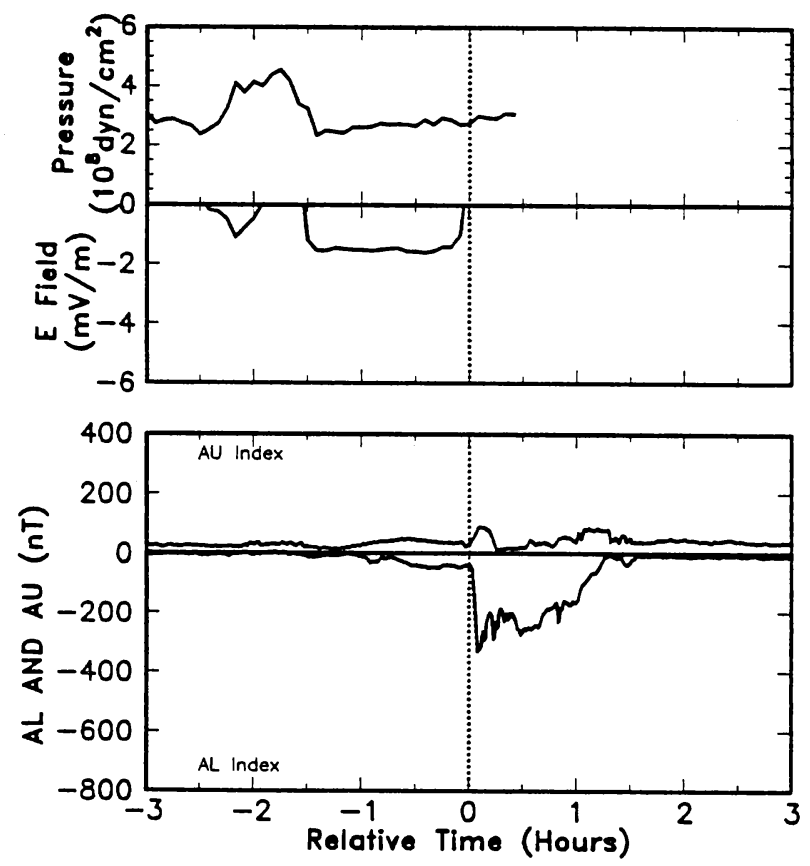

Fig. 3. Standard data plot illustrating relation between solar wind and magnetic activity. Top panel displays solar wind dynamic pressure and the rectified interplanetary electric field. Bottom panel shows the $A U$ and $A L$ indices of auroral electrojet activity. Three hours of data are plotted before and after the time of a sharp onset in the $A L$ index (vertical line). A northward turning of the IMF $B_{z}$ component (characterized by electric field returning to zero) appears to have triggered a substorm expansion phase (sudden decrease in $A L$ index). Note both IMF and pressure data are missing beginning 30 minutes after the onset.

It should be emphasized that events such as this illustrate the nonlinear nature of the solar wind interaction with the magnetosphere. For example, if we assume that magnetic activity is driven through a linear system by an increase in the southward component of the IMF, then it is impossible to explain why magnetic activity increases when the IMF turns northward. On the other hand, if we assume that the interaction produces both dissipation in the form of currents associated with magnetospheric convection and storage of energy within the magnetosphere, then it is possible to release the stored energy at a later time.

A second example of possible triggering is displayed in Fig. 4. This event differs for the previous one by a somewhat slower northward turning and by the level of activity present before the onset. Again, the activity after the onset increases for 30 minutes before it recovers to quiet values.

A third example of possible triggering is presented in Fig. 5. This event demonstrates that it is not necessary for the IMF to turn completely northward for an 


\section{COMPARISON OF SOLAR WIND INPUT WITH AURORAL ZONE MAGNETIC OUTPUT 21:38 UT February 12, 1978}

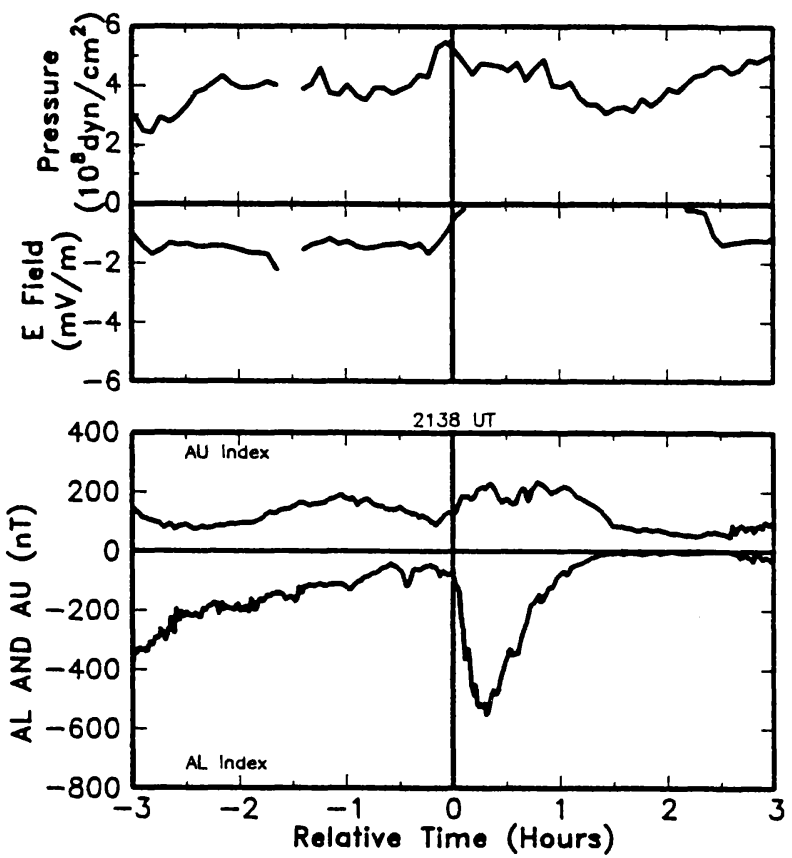

Fig. 4. A second example showing possible triggering of substorm expansion by a northward turning of the IMF.

apparent triggering to occur. There are many such events and it is difficult to decide how large a fluctuation must be to trigger an expansion onset.

\subsection{Northward turnings that do not trigger an onset in $A L$}

Northward turnings do not always trigger a substorm expansion as evident from Fig. 6. In this example, neither a northward fluctuation, nor the northward turning appear to have caused any immediate effect. Instead, about 30 minutes after the northward turning the $A L$ index began to recover, reaching quiet values in about one hour. Another example of this same behavior is presented in Fig. 7.

In both of the preceding examples the $A U$ and $A L$ indices were relatively large but constant, suggesting that the IMF was driving significant currents in the auroral oval. In neither example is there any evidence that a global substorm expansion phase occurred in the preceding three hours. In contrast to the three events discussed in the previous section, these events appear to be mainly driven by the IMF. Such events as these have previously been noted and named "convection bays" (PYTTE et al., 1978), emphasizing their interpretation as having been caused only by the convection component of the electrojets. It takes about an hour for the disturbance to decay, suggesting that release of the stored energy also takes place. 


\section{COMPARISON OF SOLAR WIND INPUT WITH AURORAL ZONE MAGNETIC OUTPUT 17:00 UT April 4, 1978}
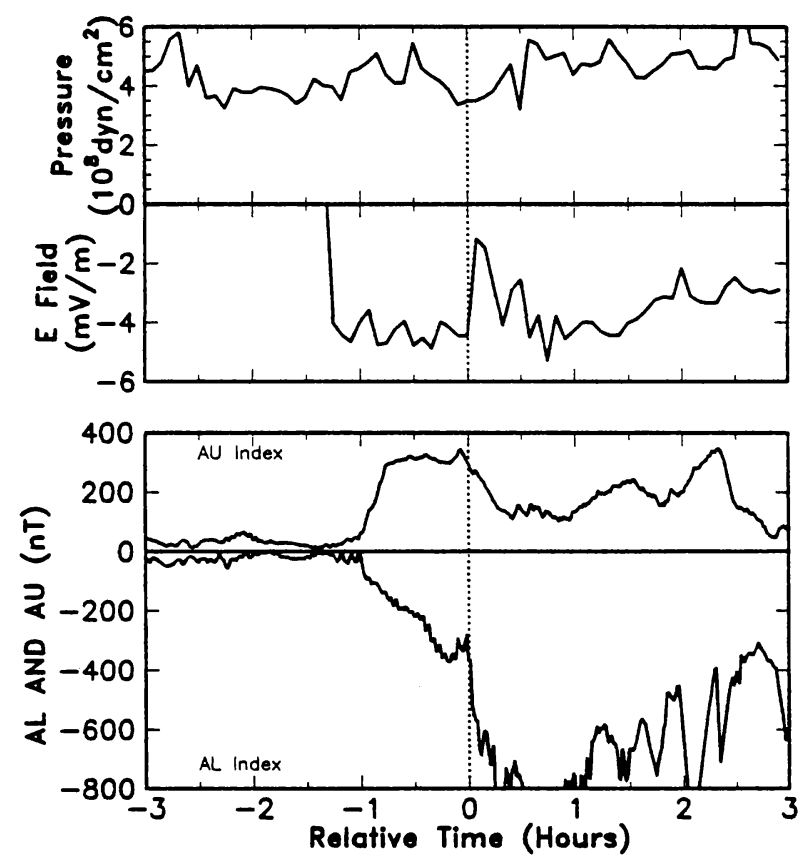

Fig. 5. A third example illustrating possible substorm triggering by a northward fluctuation in IMF $B_{z}$.

\subsection{Events with no evident solar wind trigger}

The third relevant category of relationship of the solar wind to magnetic activity is illustrated in Fig. 8. In this example, the IMF was steadily southward at a time of sudden increase in the $A L$ index. Unfortunately, the solar wind dynamic pressure began to increase slowly at this time and it is not possible to rule this out as the cause of the onset. In previous studies, however, it has been noted that relatively large, sudden increases are required to trigger onsets and, in our opinion, this is probably not the cause for this event.

A second example of onset without northward turning is presented in Fig. 9. In this case the IMF is steadily southward at the time of the onset. Again, unfortunately, there are no pressure data. We have examined midlatitude magnetograms from the day side, however, and find no conclusive evidence there for a global sudden impulse. A third example is given in Fig. 10. Again a very sharp onset occurs at the time of southward field and, in this case, steady solar wind dynamic pressure. In this example, however, the IMF is fluctuating and it is not possible to prove that the small changes shown were not the cause of the onset.

Figure 10 shows another important fact. After the sharp onset in $A L$ and its subsequent increase in magnitude, the IMF remained southward and the $A L$ index did not return to quiet values. Instead, it returned to a steady, intermediate value as in 


\section{COMPARISON OF SOLAR WIND INPUT WITH AURORAL ZONE MAGNETIC OUTPUT 22:25 UT March 22, 1978}

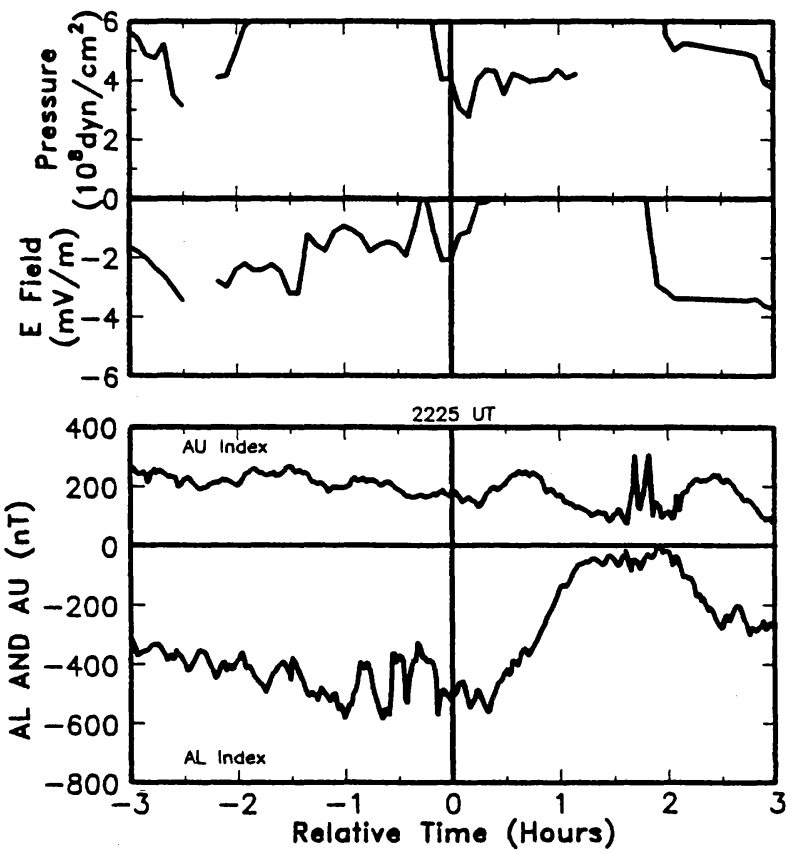

Fig. 6. A northward turning of the IMF that apparently did not trigger a substorm expansion onset. The recovery in $A L$ index is delayed approximately 30 minutes from time of northward turning (vertical line).

the case of the convection bays discussed earlier. It is interesting to speculate whether this is the normal means of initiating such bays. The implications of such a possibility are discussed later.

\subsection{Role of the azimuthal component $B_{y}$ on substorm triggering}

In an accompanying paper by TROSCHICHEV et al. (1986) it is reported that the azimuthal component of the IMF can have significant effects on the $A L$ index. One such effect occurs during winter season and causes an enhancement of $A L$ when $B_{y}$ changes from positive to negative at a time of southward $B_{z}$. In at least one case a very large change in $B_{y}$ caused an $A L$ enhancement despite $B_{z}$ being northward. Since we did not record $B_{y}$ changes, we cannot comment on this observation. According to these authors, $B_{y}$ also influences the probability of triggering by a northward turning. Turnings that occur in winter, and when $B_{y}$ is negative, are more likely to trigger onsets than those when $B_{y}$ is positive. In our analysis we originally selected 40 events for detailed plotting. Of these, six corresponded to possible trigger events. In four of these six events, $B_{y}$ was negative at the time of the onset. For the other two events $B_{y}$ was near zero. 


\section{COMPARISON OF SOLAR WIND INPUT WITH AURORAL ZONE MAGNETIC OUTPUT 15:35 UT March 18, 1978}

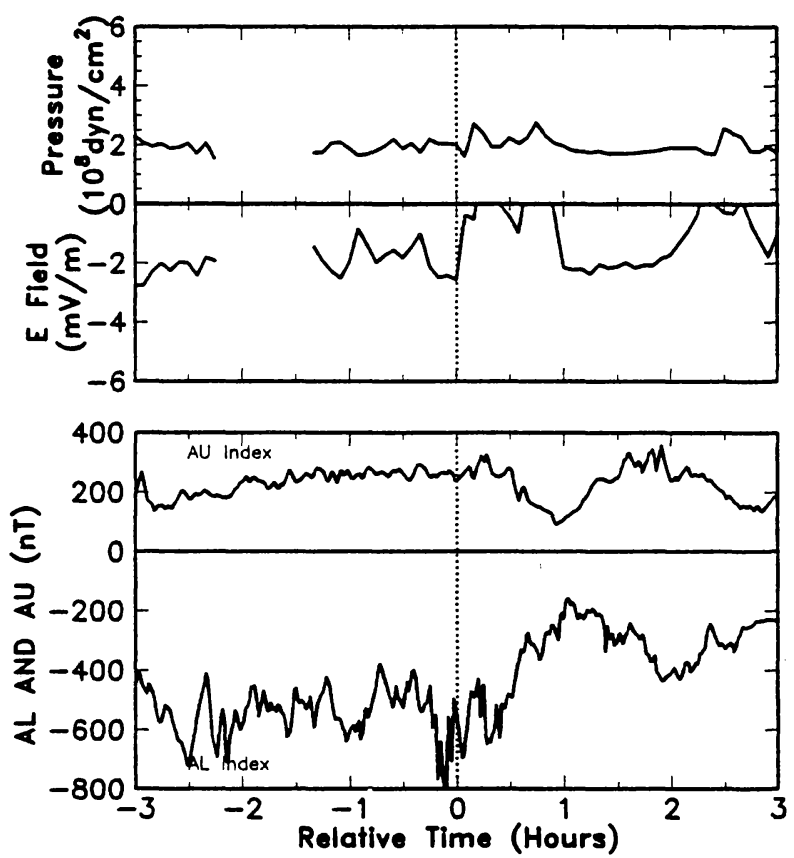

Fig. 7. A second example of northward turning without obvious substorm expansion. Note that this and the preceding figure are examples of "convection bay" activity (steady $A U$ and $A L$ indices driven by constant IMF). Not all events of this type terminate in this manner as evident from Fig. 4.

An example of a possibly triggered event when $B_{y}$ is negative is presented in Fig. 11. The top panel displays plots of the $B_{y}$ and $B_{z}$ components for the triggered onset discussed in Fig. 4. Clearly, $B_{y}$ is relatively constant and negative. On the other hand, the bottom panel displays an onset at 1210 UT, April 3, 1978 where there were no evident changes in the IMF (also see Fig. 8.) In this case $B_{y}$ was positive and relatively steady. For five selected events which had no obvious IMF trigger, $B_{y}$ was negative for two, made a negative to positive transition for one, was near zero for another, and for only one case was it positive. These observations tend to support the report of TROSCHICHEV et al. (1986), that the triggering probability is enhanced in the presence of a negative $B_{y}$ component.

\section{Statistical Summary}

Table 1 lists the results of our scan for substorm expansion onsets triggered by northward turnings of the IMF. The entries in this table represent hours in a given month when a specified event occurred in the data. The criteria for selection of events were discussed earlier, where we pointed out that many of the values are under- 


\section{COMPARISON OF SOLAR WIND INPUT WITH AURORAL ZONE MAGNETIC OUTPUT 12:10 UT April 3, 1978}

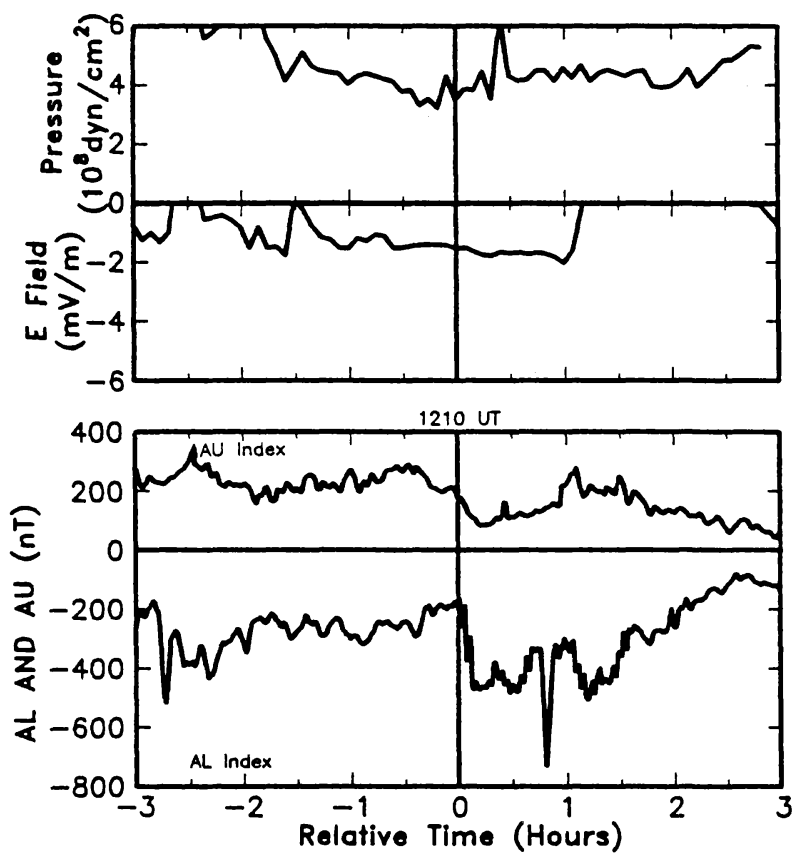

Fig. 8. A substorm expansion which is apparently not triggered by the solar wind since IMF $B_{z}$ is constant and the dynamic pressure change follows the onset.

estimates. For example, the number of substorms would probably be larger if we had a more precise definition of substorm. Similarly, the number of onsets would be greater if we had a more accurate measure of activity in the premidnight sector than $A L$.

A graphical summary of the data in Table 1 is presented in Figs. 12-14. Figure 12 is a pie chart indicating the types of magnetic activity observed in our scan of the $A L$ index. It shows that magnetic activity in the post midnight auroral oval is present about $73 \%$ of the time. This activity can be divided into three main categories, including disturbed intervals with no obvious substorm (56\%), substorms with no obvious onsets (8\%), and substorms with sharp $A L$ onsets (9\%). Clearly substorms with obvious onsets in $A L$ occur infrequently. As we show next, it is only a subset of these which appears to be triggered by the solar wind.

The relation of substorm activity to the solar wind is summarized in the pie chart plotted in Fig. 13. In this figure we consider only that fraction of the disturbed hours designated as substorm activity. According to the preceding figure this constitutes roughly $17 \%$ of the total hours scanned. As evident from Fig. 13, $47 \%$ of the substorms had no obvious sharp onset. We did not examine the corresponding IMF data for these events. Another $36 \%$ of substorms with sharp onsets had no corresponding IMF data and had to be dismissed from consideration. In all, only $18 \%$ 


\section{COMPARISON OF SOLAR WIND INPUT WITH AURORAL ZONE MAGNETIC OUTPUT 10:30 UT January 15,1978}
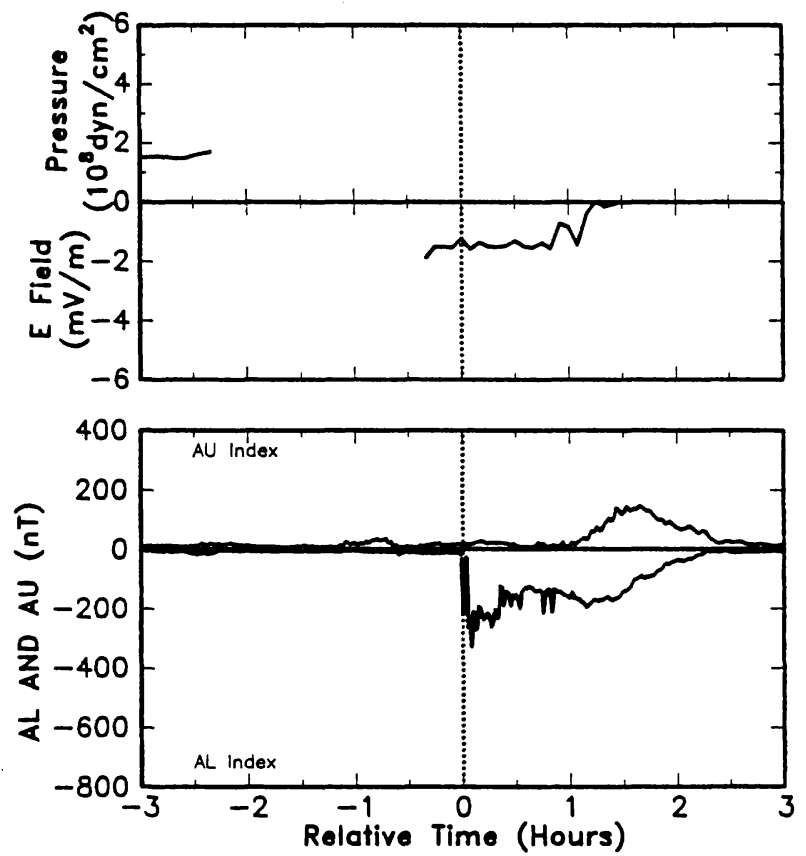

Fig. 9. A second example that which shows no change in the IMF at the time of an expansion onset. Solar wind dynamic pressure data are missing, however.

of the hours designated as substorms with sharp onsets had simultaneous IMF data. As evident from the figure, about $8 \%$ of all substorms was classified as having been possibly triggered by a northward turning. It should be emphasized, however, that it is likely that a substantial fraction of sharp onsets for which we have no solar wind data was also triggered since half of the sharp onsets for which IMF data were available appears to have been triggered. Possibly, some subset of the substorms without sharp onsets was also triggered, and the lack of obvious sharp onset is a result of a poor distribution of magnetic observatories.

The final pie chart presented in Fig. 14 considers only the hours containing substorms with sharp onset and corresponding IMF data. From this figure it can be seen that $44 \%$ of these events appeared to be triggered either by a northward turning or a northward fluctuation (not distinguished in the chart). It would thus appear that this phenomenon occurs sufficiently often to make it worthy of more detailed and careful study. By way of comparison, the well-documented phenomenon of SSC triggering (KOKUBUN et al., 1977, and references therein) occurred in only $2 \%$ of the hours scanned in our study.

Figure 14 also demonstrates another important point. The majority of all substorms with sharp onsets $(56 \%)$ cannot be correlated with obvious changes in the 


\section{COMPARISON OF SOLAR WIND INPUT WITH AURORAL ZONE MAGNETIC OUTPUT $11: 15$ UT March 31, 1978}
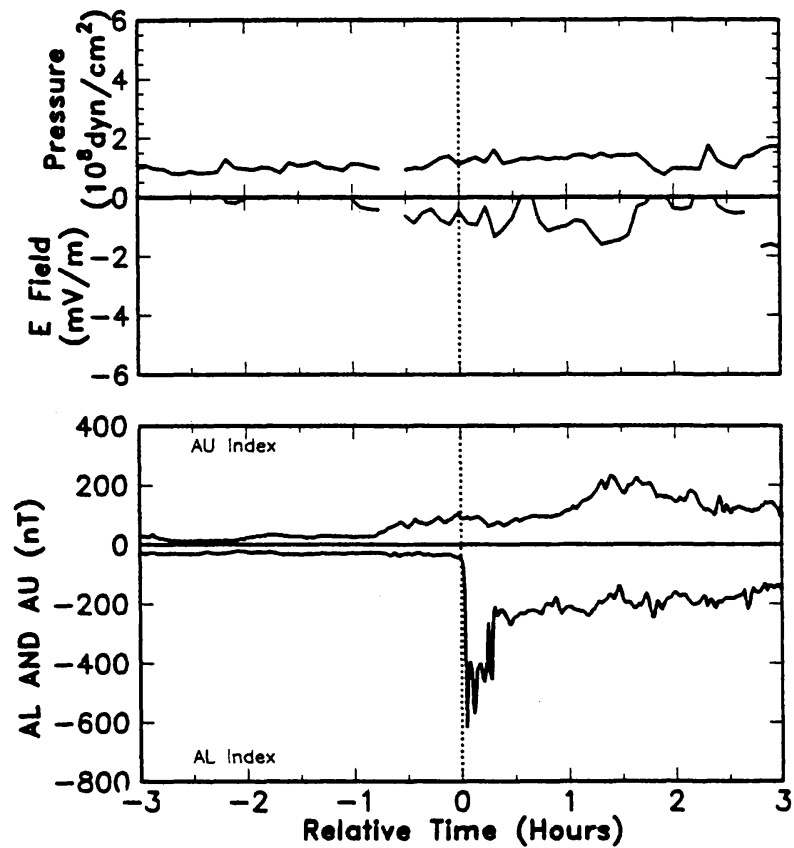

Fig. 10. A third example showing a substorm expansion with no obvious trigger. Slight fluctuations in $B_{z}$ make this example ambiguous, however.

IMF. About $45 \%$ occurs at the time of either steady southward or fluctuating southward IMF. Another $11 \%$ apparently occurs during northward IMF.

\section{Discussion}

In the preceding sections we have presented evidence that northward turnings of the IMF trigger sudden onsets of the expansion phase of magnetospheric substorms. This evidence was of two types: several case histories, and a statistical summary based on a visual scan of six months' data. Triggering by northward turnings appears to be much more common than SSC triggering which has been extensively discussed in the literature. Although triggering by northward turnings appears to occur in $44 \%$ of the cases of substorms with sharp onsets, such intervals form only a subset of all disturbed hours. About three-fourths of the disturbed hours are not easily associated with a particular substorm. Another $11 \%$ occurs during substorms with no sharp onset evident in the $A L$ index. These numbers suggest that perhaps only one eighth of all disturbed intervals contains a substorm triggered by a northward turning.

This study also demonstrated that a sudden expansion onset does not require an obvious northward turning of the IMF. While it is not impossible that a subtle, or 


\section{CHANGES IN THE IMF AT SUBSTORM ONSET}

$21: 38$ UT FEBRUARY 12, 1978

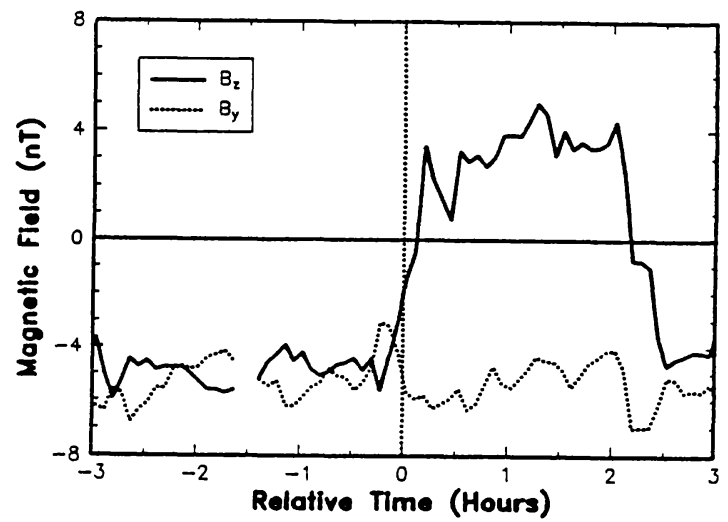

\section{CHANGES IN THE IMF AT SUBSTORM ONSET} 12:10 UT APRIL 3, 1978

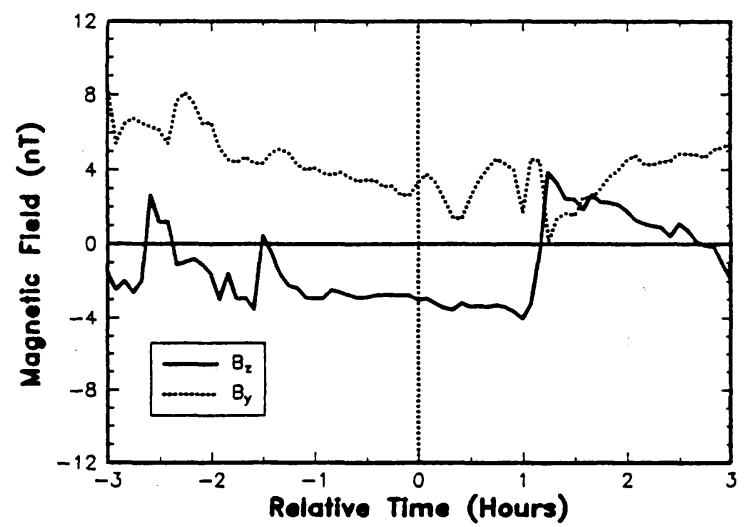

Fig. 11. Plots of the $B_{y}$ and $B_{z}$ components of the IMF in GSM coordinates. Top panel shows the data for the example of a possibly triggered onset, Fig. 4. Bottom panel shows similar data for an event with no obvious trigger, Fig. 8 . In both cases $B_{y}$ does not change significantly at the time of the onset.

unobserved change of the IMF occurred in these cases, it seems likely that the expansion onset is actually a process internal to the magnetosphere. One such process is the formation of a near-earth neutral line which releases energy stored in the magnetotail magnetic field (BAKER et al., 1984). While no accepted theoretical model of neutral line formation has been proposed, it is quite likely that it depends on the magnitude of the component of the magnetic field perpendicular to the neutral sheet (GALEEV et al., 1978). Both an SSC (storm sudden commencement) and a northward turning of the IMF could alter this component. A northward turning, for example, would be expected to reduce dayside reconnection and launch a compression wave around the earth and into the plasma sheet. Behind this wave there would be a 
Table 1. Statistics obtained in scanning six months' data in the year 1978, showing relation of auroral zone magnetic activity to the solar wind.

\begin{tabular}{lrrrrrrrrrr}
\hline & \multicolumn{10}{c}{ Month } \\
Category & Jan & Feb & Mar & Apr & May & Jun & Total & $\%$ Hrs & Fraction \\
\hline Total Hours: & 744 & 672 & 744 & 720 & 744 & 720 & 4344 & 100.0 & \\
No Disturbance & 281 & 152 & 202 & 149 & 269 & 134 & 1187 & 27.3 & .273 \\
Disturbance: & 463 & 520 & 542 & 571 & 475 & 586 & 3157 & 72.7 & \\
No Substorm & 351 & 425 & 376 & 451 & 372 & 466 & 2441 & 56.2 & .562 \\
Substorm: & 112 & 95 & 166 & 120 & 103 & 120 & 716 & 16.5 & \\
No Onset & 38 & 36 & 82 & 64 & 54 & 60 & 334 & 7.7 & .077 \\
Onset: & 74 & 59 & 84 & 56 & 49 & 60 & 382 & 8.8 & .088 \\
\hline No Data & 45 & 48 & 63 & 28 & 29 & 41 & 254 & & 1.000 \\
South & 6 & 5 & 6 & 6 & 8 & 6 & 37 & & \\
North & 8 & 0 & 2 & 0 & 1 & 4 & 15 & & \\
Turn North & 12 & 5 & 9 & 15 & 9 & 7 & 57 & & \\
Fluctuating & 2 & 1 & 4 & 7 & 1 & 2 & 17 & & \\
Pressure Inc & 1 & 0 & 0 & 0 & 1 & 0 & 2 & & \\
\hline
\end{tabular}

\section{TYPES OF AURORAL ZONE MAGNETIC ACTIVITY}

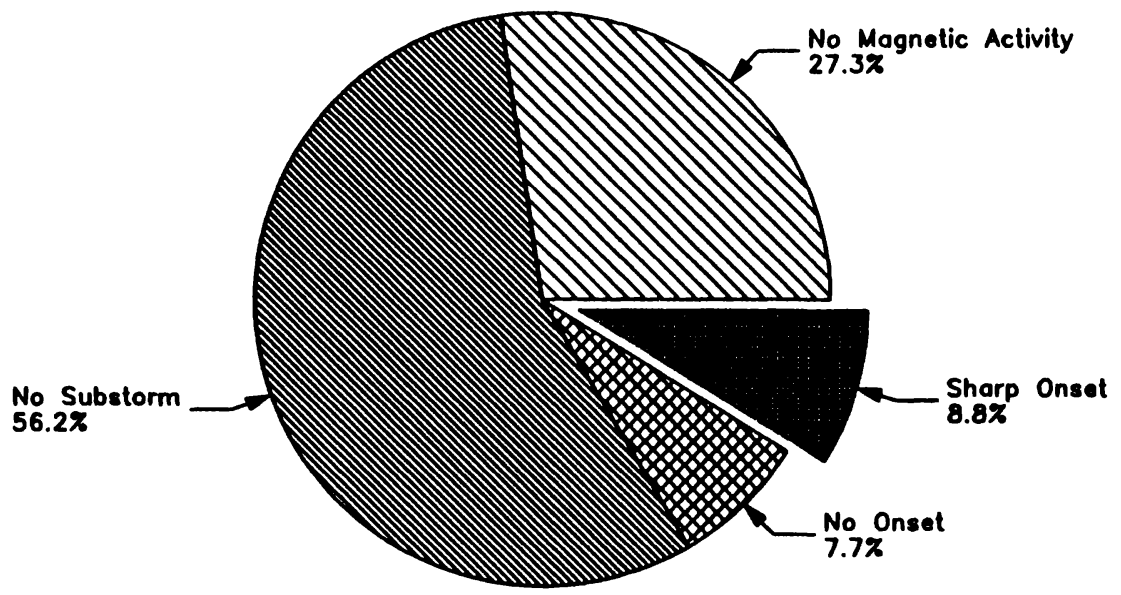

Fig. 12. A pie chart illustrating the fraction of time during the first 6 months of 1978 in which different types of magnetic activity were observed in the $A L$ index. During less than one third of the time, no magnetic activity was detectable.

reduction in earthward convective flow and an increase in magnetic field. This effect seems more likely to reduce the probability of neutral line formation than to enhance it, however. Subsequent to the northward turning, it might be expected that reconnection would occur on open field lines poleward of the polar cusps (RUSSELL, 1972; BURKE et al., 1979). This in turn should cause a reversal of the polar cap electric 


\section{RELATION OF SUBSTORMS TO SOLAR WIND}

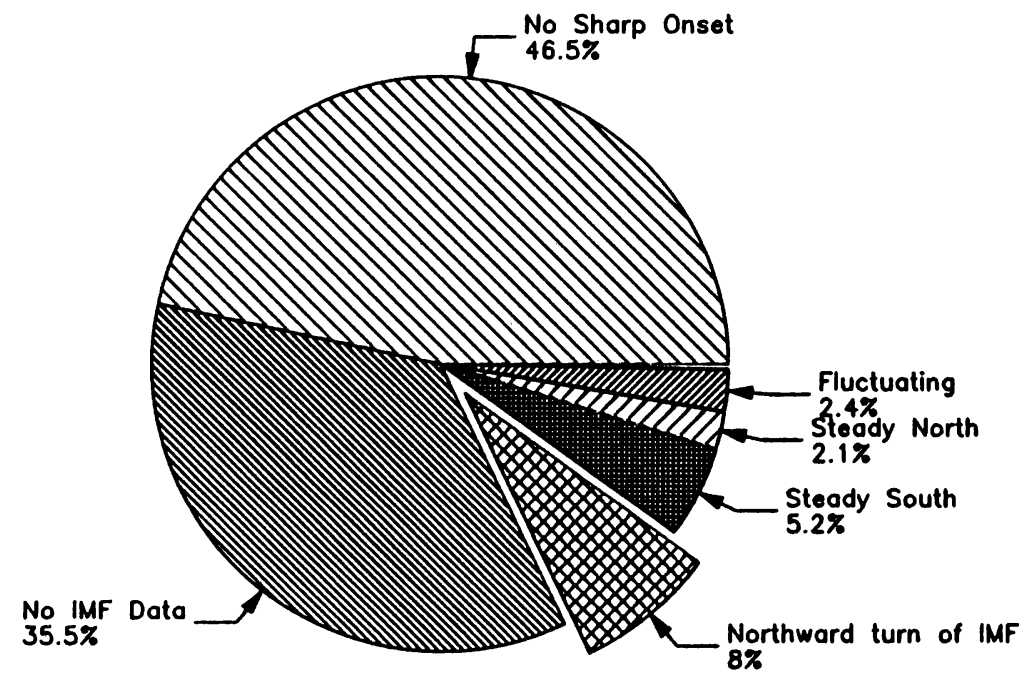

Fig. 13. A pie chart showing the fraction of time that substorm onsets were related to different features in the solar wind. Much of the time no sharp onset could be identified, although a minimum in $A L$ suggests a substorm occurred.

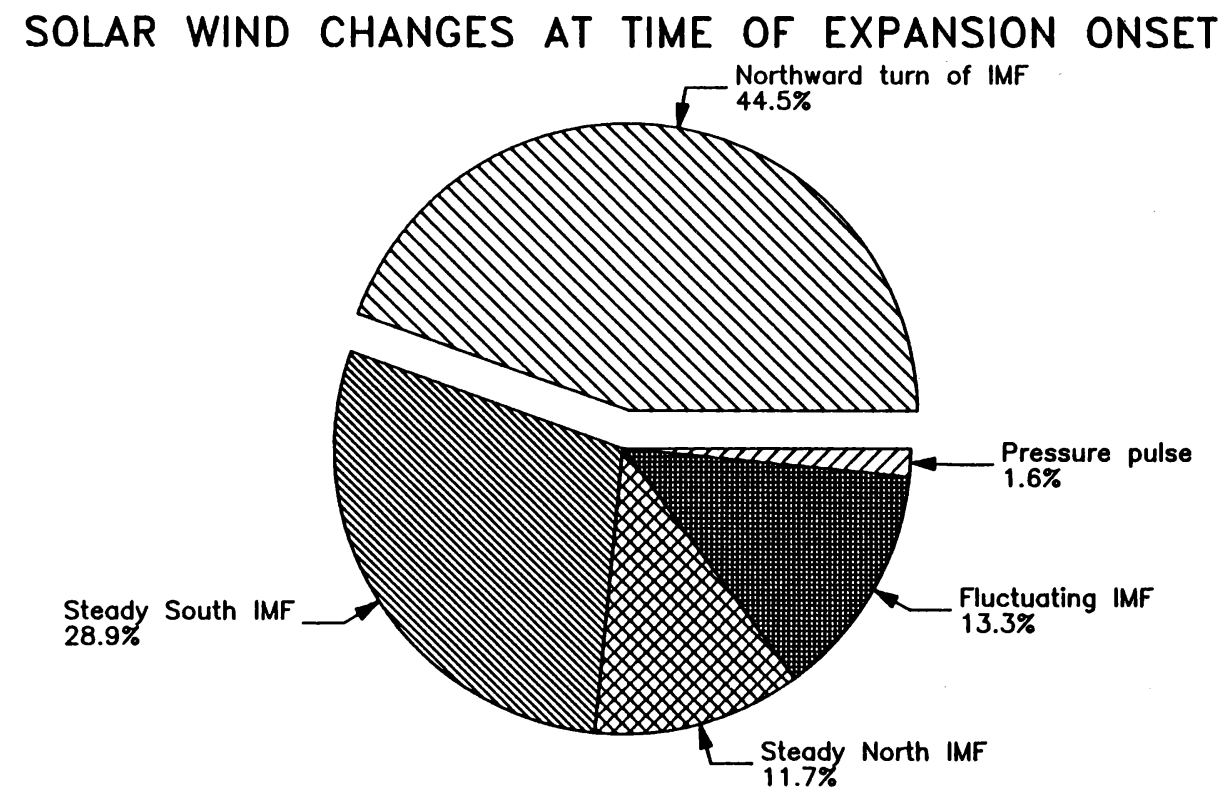

Fig. 14. Relation of sudden expansion onsets to various features of the solar wind. A substantial fraction of sudden onsets appears to be triggered. 
field and, eventually, a flow of lobe plasma away from the plasma sheet. Again, this seems likely to retard neutral line formation in the near-earth plasma sheet.

In fact the essential role of the magnetotail in substorm physics is to power substorm processes through release of stored magnetic energy. This release can be accomplished by reconnection at the neutral line which is formed temporarily in the near-earth region of the tail. However, such reconnection need not necessarily be involved in the energy release process. The energy content of the tail is reduced if the field lines move earthward and take on a less-extended configuration, regardless of whether or not the displacement involves enhanced reconnection at the temporarily formed near-earth neutral line. Following the northward turning of IMF the compression wave launched from the dayside causes earthward displacements of tail field lines as the earthward flow is weakened behind the wave while it is maintained upstream of the wave. Thus energy can be made available without the formation of the new neutral line. Indeed, it has been well established that size of the polar cap, which is considered to reflect the flux of extended, open field lines in the tail, is smaller when the IMF polarity is northward than when it is southward. The occurrence of substorms at the northward turnings of IMF could represent dissipation of the extra magnetic flux in the process of adjustment of the tail configuration to correspond to the northward IMF polarity. In this view, substorms triggered by northward IMF turnings are supported by the energy released as tail field lines assume a less extended configuration. Reconnection at the distant neutral line continues to operate until the compression wave reaches there, and hence it reduces the flux of open field lines as the supply of new open field lines from the dayside terminated when the northward IMF made contact with the dayside magnetopause.

As noted above we also identified a number of cases of northward turnings of the IMF that did not trigger a sudden onset. Several of these occurred at the end of intervals of relatively steady IMF and, incidentally, of steady $A U$ and $A L$ indices. Such intervals have previously been reported by PYTTE et al. (1978), as well as others, and have been referred to as convection bays. During such intervals most of the phenomena characteristic of expansion onset are absent. In terms of the reconnection model for substorms such steady behavior implies a balance of flux transfer. Flux returned from the nightside plasma sheet by a neutral line equals the flux eroded from the day side and transported to the tail. When the dayside erosion rate increases, enhancement of reconnection at a distant neutral line might be sufficient to return accumulating flux. In many cases, however, it appears that the convection bay is initiated by a substorm expansion, which is often associated with the formation of a near-earth neutral line. Once formed, such a neutral line might respond to minor changes in the IMF by altering the reconnection rate to balance flux transfer. In this model, events in which a northward turning occurs after a prolonged interval of southward IMF do not trigger neutral-line formation because one already exists. The observed 30-minute time delay between the northward turning and the recovery of activity may represent the time for the neutral line to retreat to its distant location and, in the process, dissipate the remaining energy stored in the magnetotail.

The work reported here was initiated while the principal author was a visiting professor at the Institute of Space and Astronautical Science in Tokyo, Japan. Work at the University of California, Los Angeles, was supported by grants from the National Science Foundation 
(ATM83-18200), the Office of Naval Research (N00014-84-C-0158), and the National Aeronautics and Space Administration (NGL-05-007-004). Solar wind data were provided by the National Space Science Data Center at Goddard Space Flight Center in Greenbelt, MD. Magnetic indices were provided by the World Data Center C 2 for Geomagnetism at Kyoto University, Kyoto, Japan. High resolution plots of IMP 8 magnetic field were produced at UCLA by Dr. C. T. Russell. We would like to thank G. Siscoe for his helpful remarks after a presentation of this work.

\section{REFERENCES}

Akasofu, S.-I., Polar and Magnetic Substorms, Reidel, Dordrecht, Holland, 1968.

Baker, D. N., S.-I. Akasofu, W. Baumjohann, J. W. Bieber, D. H. Fairfield, E. W. Hones, Jr., B. Mauk, R. L. McPherron, and T. E. Moore, Substorms in the magnetosphere, in Solar Terrestrial Physics: Present and Future, edited by D. M. Butler and K. Papadopoulos, Chapter 8, NASA Reference Pub. 1120, 1984.

Burch, J. L., Triggering of the substorm expansion phase by directional discontinuities in the interplanetary magnetic field, EOS Trans. $A G U, \mathbf{5 4}, 413,1973$.

Burke, W. J., M. C. Kelley, R. C. Sagalyn, M. Smiddy, and S. T. LaI, Polar cap electric field structures with a northward interplanetary magnetic field, Geophys. Res. Lett., 6, 21, 1979.

CAAN, M. N., R. L. MCPHERron, and C. T. RusSEll, Substorm and interplanetary magnetic field effect on the geomagnetic tail lobes, J. Geophys. Res., 80(1), 191-194, 1975.

CaAn, M. N., R. L. McPherron, and C. T. Russell, Characteristics of the association between the interplanetary magnetic field and substorms, J. Geophys. Res., 82(29), 4837-4842, 1977.

Coroniti, F. V., R. L. McPherron, and G. K. Parks, Studies of the magnetospheric substorm: 3. Concept of the magnetospheric substorm and its relation to electron precipitation and micropulsations, J. Geophys. Res., 73(5), 1715-1722, 1968.

Galeev, A. A., F. V. Coroniti, and M. Ashour-Abdalla, Explosive tearing mode reconnection in the magnetospheric tail, Geophys. Res. Lett., 5(8), 707-710, 1978.

KameI, T. and H. MAEDA, Auroral electrojet indices (AE) for January-June 1978, Data Book No. 3, Data Analysis Center for Geomagnetism and Spacemagnetism, Faculty of Science, Kyoto University, Kyoto, Japan, April 1981.

Kokubun, S., R. L. MCPherron, and C. T. Russell, Triggering substorms by solar wind discontinuities, J. Geophys. Res., 82(1), 74-86, 1977.

McPherron, R. L., Growth phase of magnetospheric substorms, J. Geophys. Res., 75(28), 5592-5599, 1970.

McPherron, R. L., Magnetospheric substorms, Rev. Geophys. Space Phys., 17(4), 657-681, 1979.

McPherron, R. L., C. T. Russell, and M. Aubry, Satellite studies of magnetospheric substorms on August 15, 1978, 9. Phenomenological model for substorms, J. Geophys. Res., 78(16), 3131-3149, 1973.

Nishida, A. and N. Nagayama, Synoptic survey for the neutral line in the magnetotail during the substorm expansion phase, J. Geophys. Res., 78(19), 3782-3798, 1973.

Pellinen, R. J., W. Baumjohann, W. Heiknila, V. A. Sergeev, A. G. Yahnin, G. Marklund, and A. O. MELNIKOV, Event study of presubstorm phases and their relation to the energy coupling between solar wind and magnetosphere, Planet. Space Sci., 30(4), 371-388, 1982.

Pytte, T., R. L. McPherron, E. W. Hones, Jr., and H. I. West, Jr., Multiple-satellite studies of magnetospheric substorms, III. Distinction between polar substorms and convection-driven negative bays, J. Geophys. Res., 83(A2), 663-679, 1978.

ROSTOKER, G., Triggering of expansive phase intensifications of magnetospheric substorms by northward turnings of the interplanetary magnetic field, J. Geophys. Res., 88(A9), 6981-6993, 1983.

Rostoker, G., S.-I. Akasofu, J. Foster, R. A. Greenwald, Y. Kamide, K. Kawasaki, A. T. Y. Lui, R. L. MCPherron, and C. T. Russell, Magnetospheric substorms-definition and signatures, $J$. Geophys. Res., 85(A4), 1663-1668, 1980.

Rostoker, G., M. Mareschal, and J. C. Samson, Response of day side net downward field-aligned current to changes in the interplanetary magnetic field and to substorm perturbations, J. Geophys. Res., 87(A5), 3489-3510, 1982. 
Rostoker, G., W. Baumjohann, and C. T. Russell, A case study of the response of the magnetosphere to changes in the interplanetary medium, J. Geophys., 53, 170-181, 1983.

Rostoker, G., I. SPADINGER, and J. C. SAMSON, Local time variations in the response of Pc 5 pulsations in the morning sector to substorm expansive phase onsets near midnight, J. Geophys. Res., 89(A8), 6749-6757, 1984.

Russell, C. T., The configuration of the magnetosphere, in Critical Problems of Magnetospheric Physics, edited by E. R. Dyer, pp. 1-16, National Academy of Sciences, Washington, D.C., 1972.

Russell, C. T. and R. L. MCPherron, The magnetotail and substorms, Space Sci. Rev., 11, 111- 222, 1972.

Troschichev, O. A., A. L. Kotikov, B. D. Bolotinskaya, and V. G. Andrezen, Influence of the IMF azimuthal component on magnetospheric substorm dynamics, this issue, 1986. 